\title{
The role of myocardial performance index and Nt-proBNP levels as a marker of heart dysfunction in nonalcoholic cirrhotic patients
}

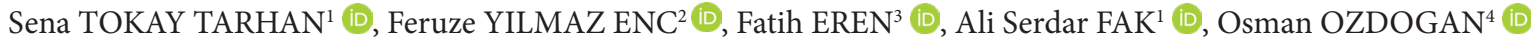 \\ ${ }^{1}$ Department of Internal Medicine, School of Medicine, Marmara University, Pendik, Istanbul, Turkey. \\ ${ }^{2}$ Department of Gastroenterology, School of Medicine, Medeniyet University, Goztepe, Istanbul, Turkey. \\ ${ }^{3}$ Institute of Gastroenterology, Marmara University, Maltepe, Istanbul, Turkey. \\ ${ }^{4}$ Department of Gastroenterology, School of Medicine, Marmara University, Pendik, Istanbul, Turkey.
}

Corresponding Author: Sena TOKAY TARHAN

E-mail: senatokay@hotmail.com

Submitted: 03.07.2021 Accepted: 25.10.2021

\begin{abstract}
Objective: Cardiac dysfunction has been reported in both cirrhotic and alcoholic patients. Our aim was to determine the relation of serum $\mathrm{N}$-terminal pro-B-type natriuretic peptide (Nt-proBNP) levels to myocardial performance index (MPI) and disease severity in nonalcoholic cirrhotic patients.

Patients and Methods: In this prospective study including 25 cirrhotic patients and 27 healthy controls, MPI was assessed by pulsedwave tissue Doppler imaging (PW-TDI). The disease severity was determined by Child-Turcotte-Pugh (CTP ) and model for endstage liver disease (MELD) scores.

Results: There were no statistically significant differences in MPI levels between patients and controls $(\mathrm{p}<0.246)$. Nt-proBNP levels $(\mathrm{p}<0.0003)$, cardiac output $(\mathrm{CO})(\mathrm{p}<0.0002)$, left ventricular end-systolic (LVES) volume $(\mathrm{p}<0.031)$ and $\mathrm{QT}$ interval $(\mathrm{p}<0.0001)$ increased and left ventricular systolic function was normal in all cirrhotic patients when compared to controls. Nt-proBNP levels were positively correlated with MELD scores $(\mathrm{p}<0.0001, \mathrm{r}=0.59)$, QT duration $(\mathrm{p}<0.0001, \mathrm{r}=0.59), \mathrm{CO}(\mathrm{p}=0,001, \mathrm{r}=0.44)$, right atrial $(\mathrm{RA})$ area $(\mathrm{p}=0.026, \mathrm{r}=0.31)$ and negatively correlated with diastolic $\mathrm{BP}(\mathrm{p}=0.015, \mathrm{r}=-0.34)$.

Conclusion: We conclude that in nonalcoholic cirrhotic patients, left ventricular MPI and systolic function were normal. Nt-proBNP levels were correlated with the disease severity and hyperdynamic circulation.

Keywords: Cirrhosis, Heart failure, Nt-proBNP, Echocardiography
\end{abstract}

\section{INTRODUCTION}

Cirrhosis is characterized by hyperdynamic circulation with increased cardiac output, heart rate and a decreased systemic vascular resistance. Impaired cardiac contractility and performance in patients with cirrhosis has been termed 'cirrhotic cardiomyopathy' [1].

Alcohol is toxic to many organs including the liver and heart. Chronic alcohol abuse may induce hypertrophy, apoptosis and necrosis of myocytes and leads to both systolic and diastolic cardiac dysfunction and ultimately left and right ventricular dilatation [2].

Brain natriuretic peptide (BNP) which is secreted from ventricles in response to pressure and volume overload is a sensitive marker for heart disease. It relaxes vascular smooth muscle, causes arterial and venous dilation and leads to reduced blood pressure. It is secreted as pre-pro-BNP which is subseqently cleaved to proBNP, N-terminal pro-B-type natriuretic peptide (Nt-proBNP) and biologically active BNP [3]. Increased levels of BNP are related to diastolic cardiac dysfunction [4-6] and to the severity of the disease in patients with cirrhosis [4, 7-10]. High level of BNP is an independent predictor of mortality in cirrhotic patients $[9,11]$.

Myocardial performance index (MPI) described first by Tei and his co-workers is a new Doppler-derived index reflecting systolic and diastolic function of the heart. This simple and reproducible technique is not affected by heart rate, blood pressure, ventricular loading conditions [12]. It can be measured either by pulsedwave Doppler (PWD) or pulsed-wave tissue Doppler imaging (PW-TDI). Both methods have high diagnostic accuracy for congestive heart failure, but conventional PWD may be affected by heart rate variability [13].

There have been few studies investigating the role of MPI in cirrhotic patients [10, 14-17].

How to cite this article: Tahan Tokay S, Enc Yilmaz F, Eren F, Fak AS, Ozdogan O. The role of myocardial performance index and Nt-proBNP levels as a marker of heart dysfunction in nonalcoholic cirrhotic patients. Marmara Med J 2022; 35(1):10-16. doi: 10.5472/marumj.1056204 
The aim of this study was to investigate the role of both MPI, measured by PW-TDI and Nt-proBNP levels on the assessment of cardiac function in nonalcoholic cirrhotic patients.

\section{PATIENTS and METHODS}

The study was designed as a prospective, clinical study involving the analysis of Nt-proBNP levels and echocardiographic evaluation of MPI in cirrhotic patients and controls. Twenty-five patients with liver cirrhosis and 27 controls were enrolled in the study. Diagnosis of cirrhosis was based on clinical, biochemical, endoscopic and ultrasonographic criteria.

Patients suffering from diseases potentially associated with high BNP levels including heart failure unrelated to liver disease, arterial hypertension, renal failure, respiratory failure, obesity (body mass index $>30 \mathrm{~kg} / \mathrm{m}^{2}$ ), diabetes, severe anemia $(\mathrm{Hg}<7 \mathrm{~g} / \mathrm{dL})$, were excluded from the study. Patients with electrocardiographic and with echocardiographic evidence of structural heart disease and patients with alcohol dependence were also excluded from the study. All patients had normal cardiac physical examination and were in sinus rhythm at admission. Patients with bundle branch block, atrioventricular block, atrial fibrillation and pacemaker rhythm on electrocardiography were excluded from the study.

Patients were classified according to their Child-TurcottePugh (CTP) scores as Stage A, B and C [17], then model for end-stage liver disease (MELD) scores were calculated. Stage A patients were compensated and Stage B and C patients were decompensated patients.

\section{Echocardiographic Assessment}

All echocardiographic measurements were performed according to the recommendations of the American Society of Echocardiography $[18,19]$. M-mode, two-dimensional echocardiography, pulsed wave Doppler, continuous-wave Doppler and tissue Doppler studies were performed using a commercially available echocardiograph operating at 2,5-3 MHz.

\section{Two-Dimensional Doppler Echocardiography}

M-mode images were used for the measurement of left ventricular (LV) dimensions at end - diastole in the parasternal long-axis view. Left ventricular ejection fraction (LVEF) and left ventricular volumes were assessed by the biplane method of disks (modified Simpson's rule). The left ventricular mass (LVM) was calculated according to Cube formula and LVM index (LVMi) was defined as the ratio between LVM and body surface area. Left atrial volume (LAV) was calculated by arealength technique and was indexed to body surface area (LAVi). By planimetric method right atrial (RA) area was measured in the apical 4-chamber view at end-systole just prior to tricuspid valve opening and the area under the tricuspid valve annulus was excluded. Right ventricular (RV) area was measured by manually tracing RV endocardial border from the lateral tricuspid annulus to the apex and back to medial tricuspid annulus at end-diastole in RV focused apical 4-chamber view.
Papillary muscles, moderator band and trabeculations were included in the cavity area.

Pulsed-wave Doppler examination of mitral inflow was performed in the apical 4-chamber position during quiet respiration by placing the Doppler sample volume in the middle of the LV inflow tract $1 \mathrm{~cm}$ below the plane of mitral annulus between the mitral leaflet tips. Maximal flow velocity in early diastole was recorded and velocity of the mitral inflow early, rapid-filling wave $(\mathrm{E})$, the peak velocity of the late filling wave due to atrial contraction (A), and the E/A ratio were obtained.

Cardiac output (CO) was calculated as Doppler estimated time velocity integral (TVI) of the aortic systolic flow multiplied by cross-sectional area of the aorta and the heart rate. The aortic TVI was recorded from the apical 5 -chamber view by placing the continuous-wave Doppler sample volume at the level of the aortic annulus, approximately 3-5 mm proximal to the valve. The diameter of the aortic annulus (D) was measured by recording the parasternal long-axis view in systole and CO was calculated using the following formula: $\mathrm{CO}=0.785 \times \mathrm{D}^{2} \times \mathrm{TVI} \times$ heart rate [20].

\section{Pulsed - Wave Tissue Doppler Imaging}

Pulsed-wave tissue Doppler imaging was performed by placing the sample volume at the lateral corner of the mitral annulus from the apical 4-chamber view during quiet respiration. To display tissue velocities the gain was minimized and the wall filter settings were adjusted to exclude high frequency signals. Annular early (e') and late (a') diastolic velocities were measured. The transmitral E/e' ratio was calculated. To determine the MPI, the time interval from the end to the onset of the mitral annular velocity pattern during diastole (a) and the duration of the S-wave (b) were measured. MPI was calculated as (a-b)/b [21].

Left ventricular diastolic dysfunction (LVDD) was classified into four categories as: normal diastolic function $\mathrm{E} / \mathrm{A}>0.75$ and $\mathrm{E} / \mathrm{e}^{\prime}$ $<10$; mild LVDD, E/A $\leq 0.75$ and $\mathrm{E} / \mathrm{e}^{\prime}<10$; moderate LVDD, E/A $\leq 1.5$ and $\mathrm{E} / \mathrm{e}^{\prime} \geq 10$; severe $\mathrm{LVDD}, \mathrm{E} / \mathrm{A}>1.5$ and $\mathrm{E} / \mathrm{e}^{\prime} \geq 10$ [22].

All measurements were averaged from three consecutive cardiac cycles and an investigator blinded to all data performed the echocardiographic and electrocardiographic analyses.

Resting 12-lead surface ECG was obtained from all subjects at a speed of $25 \mathrm{~mm} / \mathrm{s}$ with an electrocardiograph. QT interval was calculated manually by measuring the interval from the onset of the QRS complex to the end of the T wave. Three consecutive Q-T intervals were measured in each lead and were averaged. If a $U$ wave was present, the Q-T interval was measured from the onset of QRS complex to the nadir of the curve between the $T$ and $U$ waves.

\section{Biochemical Assays}

In all patients venous blood samples were drawn into ethylenediaminetetraacetic acid (EDTA) containing tubes and plasma was removed by centrifugation and was frozen at -80 $\mathrm{C}$ before analysis. Nt-proBNP serum level was estimated using an electrochemiluminescent immunoassay kit - (analisator MODULAR ANALITICS E 170, Roche Diagnostics GmbH, D-68296 Mannheim, Germany). Measurement range was of 5-35000 pg/ml. Normal values were $<125 \mathrm{pg} / \mathrm{ml}$. Peripheral 
venous blood samples were used for all estimations and biochemical analyses were performed using standard methods.

The study was approved by the Clinical Research Ethics Committee of Marmara University, School of Medicine, Istanbul (03 - 2006-0124), and informed written consent was obtained from all the participants.

\section{Statistics Analysis}

Statistical analyses were performed using Prism software (GraphPad Prism 6.0, USA; serial no. GPM6-131891-RJJW4325E). Descriptive data were presented as percentage and mean \pm standard deviation. The Mann-Whitney $U$ test was used to compare continuous variables in two independent groups and Kruskal-Wallis test was used to compare continuous variables in three independent groups. Dunn's multiple comparison test was used for post hoc analyses. Spearman's correlation was used to assess the correlation of continuous variables. A p value $<0.05$ was considered as statistically significant.

\section{RESULTS}

The study group comprised 25 patients, 10 women and 15 men with an average age of $47.88 \pm 14.34$ years. The control group included 27 healthy participants, 13 women and 14 men with an average age of $43.88 \pm 8.41$ years. The causes of cirrhosis were hepatitis $B$ virus infection-associated post-hepatic cirrhosis in 13 patients, hepatitis $C$ virus infection in 6 , and cryptogenic cirrhosis in 6 patients. Among them, 5 patients exhibited ascites, 4 experienced gastrointestinal bleeding, 1 had encephalopathy, 2 experienced spontaneous bacterial peritonitis, and 12 patients had a history of propranolol treatment. The cirrhotic patients were categorized as A $(n=15), B(n=6)$ or C $(n=4)$ according to their CTP scores. Demographic, clinical and biochemical characteristic of the cirrhotic patients and control groups are given in Table I. Plasma Nt-proBNP levels of cirrhotic patients were found to be significantly elevated than those of control participants (63.92 \pm 18.45 vs $45.74 \pm 8.62, \mathrm{p}<0.0003)$. Mean MELD score, aspartate aminotransferase, alanine aminotransferase, international normalized ratio, total and direct bilirubin levels in cirrhotic patients were also significantly higher than those in controls $(\mathrm{p}<0.05)$. Albumin, hemoglobin, thrombocyte, and leukocyte values in cirrhotic patients were significantly lower than those in controls $(\mathrm{p}<0.05)$ (Table I).

Echocardiographic and electrocardiographic parameters of cirrhotic patients and controls are shown in Table II. There was no statistically significant difference in MPI levels between patients and of controls ( $p>0.05$ ). Only left ventricular endsystolic (LVES) volume and CO were higher in cirrhotic patients than in controls $(\mathrm{p}<0.05)$. Left ventricular end-diastolic (LVED) volume and LVMi were also higher in cirrhotic patients compared to controls, but the differences were not statistically significant. Additionally, QT intervals were also higher in cirrhotic patients $(\mathrm{p}<0.05)$. (Table II).

Clinical,biochemical, echocardiographicand electrocardiographic parameters of compensated and decompensated patients and controls are presented in Table III. In post-hoc analysis Nt-proBNP levels, CO, LAVi, LVED volume and QT interval of decompensated patients were significantly higher than those of controls $(\mathrm{p}<0.05)$. Additionally, LAVi and E/e' ratio were significantly higher in decompensated patients than in compensated patients $(\mathrm{p}<0.05)$. Diastolic BP was lower in decompensated patients compared to controls $(\mathrm{p}<0.05)$. (Table III).

Table I. Demographic, clinical and biochemical parameters in cirrhotic $(n=25)$ patients and controls $(n=27)$

\begin{tabular}{|c|c|c|c|}
\hline Characteristics & $\begin{array}{c}\text { Cirrhosis } \\
(\mathrm{n}=25)\end{array}$ & Control $(n=27)$ & $\mathbf{p}^{*}$ \\
\hline Age & $47.88 \pm 14.34$ & $43.88 \pm 8.41$ & 0.387 \\
\hline Systolic blood pressure $(\mathrm{mmHg})$ & $120.60 \pm 10.83$ & $122.70 \pm 8.27$ & 0.365 \\
\hline Diastolic blood pressure (mmHg) & $76.00 \pm 5.00$ & $78.46 \pm 3.68$ & 0.053 \\
\hline Pulse (beats/min) & $72.32 \pm 10.46$ & $68.96 \pm 9.62$ & 0.325 \\
\hline MELD score & $12.17 \pm 4.43$ & $6.35 \pm 056$ & $<0.0001$ \\
\hline Nt-proBNP (pg/ml) & $63.92 \pm 18.45$ & $45.74 \pm 8.62$ & $<0.0003$ \\
\hline Sodium $(\mathrm{mEq} / \mathrm{L})$ & $138.2 \pm 4.22$ & $139.9 \pm 2.71$ & 0.071 \\
\hline Creatinine $(\mathrm{mg} / \mathrm{dL})$ & $0.82 \pm 0.22$ & $0.88 \pm 0.14$ & 0.188 \\
\hline Hemoglobin (gr/dL) & $12.56 \pm 1.68$ & $13.62 \pm 1.50$ & 0.024 \\
\hline Trombocyte $\left(10^{3} / \mu \mathrm{L}\right)$ & $76.264 \pm 28.87$ & $289.07 \pm 69.49$ & $<0.0001$ \\
\hline Leukocyte $\left(10^{3} / \mu \mathrm{L}\right)$ & $3.989 \pm 1.457$ & $6.100 \pm 1.359$ & $<0.0001$ \\
\hline Aspartate Aminotransferase (U/L) & $53.24 \pm 24.01$ & $30.73 \pm 5.05$ & $<0.0001$ \\
\hline Alanine Aminotransferase (U/L) & $50.36 \pm 35.59$ & $32.81 \pm 6.69$ & 0.032 \\
\hline Total Bilirubin (mg/dL) & $1.94 \pm 1.36$ & $0.63 \pm 0.18$ & $<0.0001$ \\
\hline Direct Bilirubin (mg/dL) & $0.66 \pm 0.68$ & $0.20 \pm 0.17$ & $<0.0001$ \\
\hline Albumin $(\mathrm{gr} / \mathrm{dL})$ & $3.72 \pm 0.73$ & $4.60 \pm 0.47$ & $<0.0001$ \\
\hline International Normalized Ratio & $1.38 \pm 0.31$ & $0.96 \pm 0.10$ & $<0.0001$ \\
\hline
\end{tabular}

Table II. Echocardiographic and electrocardiographic parameters of cirrhotic patients and controls

\begin{tabular}{|c|c|c|c|}
\hline Parameters & Cirrhosis $(\mathrm{n}=25)$ & Control $(n=27)$ & $\mathbf{p}^{*}$ \\
\hline MPI & $0.32 \pm 0.09$ & $0.27 \pm 0.11$ & 0.246 \\
\hline $\operatorname{LAVi}\left(\mathrm{ml} / \mathrm{m}^{2}\right)$ & $29.80 \pm 10.55$ & $23.57 \pm 3.79$ & 0.055 \\
\hline RA area $\left(\mathrm{cm}^{2}\right)$ & $16.37 \pm 4.14$ & $14.27 \pm 2.56$ & 0.116 \\
\hline $\mathrm{RV}$ area $\left(\mathrm{cm}^{2}\right)$ & $24.37 \pm 3.58$ & $24.37 \pm 3.58$ & 0.678 \\
\hline IVS (mm) & $9.67 \pm 1.34$ & $9.07 \pm 2.09$ & 0.350 \\
\hline PW (mm) & $9.31 \pm 1.31$ & $8.59 \pm 1.98$ & 0.186 \\
\hline LVMi $\left(\mathrm{gr} / \mathrm{m}^{2}\right)$ & $101.00 \pm 19.71$ & $93.23 \pm 25.11$ & 0.057 \\
\hline LVED volume $(\mathrm{ml})$ & $118.80 \pm 34.95$ & $99.54 \pm 19.46$ & 0.055 \\
\hline LVES volume (ml) & $46.58 \pm 16.03$ & $38.21 \pm 10.47$ & 0.031 \\
\hline LVEF \% & $61.60 \pm 6.97$ & $61.81 \pm 5.99$ & 0.777 \\
\hline $\mathrm{CO}(\mathrm{L} / \mathrm{min})$ & $5.57 \pm 1.24$ & $4.32 \pm 0.71$ & 0.0002 \\
\hline $\mathrm{E} / \mathrm{A}$ & $1.34 \pm 0.35$ & $1.38 \pm 0.33$ & 0.784 \\
\hline $\mathrm{e}^{\prime}(\mathrm{cm} / \mathrm{s})$ lateral mitral annulus & $12.40 \pm 2.49$ & $12.63 \pm 2.66$ & 0.993 \\
\hline E/e' & $6.95 \pm 2.05$ & $6.38 \pm 1.56$ & 0.398 \\
\hline QT interval (msec) & $424.90 \pm 22.93$ & $396.20 \pm 16.58$ & 0.0001 \\
\hline
\end{tabular}

${ }^{*}$ Mann-Whitney $U$ test, LAVi: left atrial volume index, RA: right atrium, RV: right ventricle, IVS: interventricular septum, PW: posterior wall, LVMi: left ventricular mass index, LVED: left ventricular end-diastolic, LVES: left ventricular end-systolic, LVEF: left ventricular ejection fraction, CO: cardiac output, MPI: myocardial performance index 
Left ventricular systolic function was normal in all cirrhotic patients, but one patient (CTP A) exhibited mild, one patient (CTP B) exhibited moderate and one patient (CTP B) exhibited severe diastolic dysfunction.

Plasma Nt-proBNP levels were positively correlated with MELD scores $(\mathrm{p}<0.0001, \mathrm{r}=0.59)$, QT duration $(\mathrm{p}<0.0001, \mathrm{r}=0.59)$, CO $(\mathrm{p}=0.001, \mathrm{r}=0.44)$, RA area $(\mathrm{p}=0.026, \mathrm{r}=0.31)$ and were negatively correlated with diastolic $B P(p=0.015, r=-0.34)$.

Table III. Clinical, echocardiographic and electrocardiographic parameters of cirrhotic patients and controls according to Child-TurcottePugh score

\begin{tabular}{|c|c|c|c|c|}
\hline Parameters & $\begin{array}{c}\text { CTP Stage A } \\
\quad(n=15)\end{array}$ & $\begin{array}{l}\text { CTP Stage } \\
B+C(n=10)\end{array}$ & $\begin{array}{l}\text { Control } \\
(\mathrm{n}=27)\end{array}$ & $\mathbf{p}^{*}$ \\
\hline $\begin{array}{l}\text { Systolic blood } \\
\text { pressure }(\mathrm{mmHg})\end{array}$ & $120.70 \pm 9.17$ & $120.50 \pm 13.13$ & $122.70 \pm 8.27$ & 0.657 \\
\hline $\begin{array}{l}\text { Diastolic blood } \\
\text { pressure(mmHg) }\end{array}$ & $77.86 \pm 4.26$ & $73.64 \pm 5.05$ & $78.46 \pm 3.68$ & 0.010 \\
\hline MELD score ${ }^{*}, *$ & $9.69 \pm 2.56$ & $15.09 \pm 4.46$ & $6.35 \pm 0.56$ & $<0.0001$ \\
\hline $\begin{array}{l}\text { Nt-proBNP (pg/ } \\
\text { ml)* }\end{array}$ & $58.72 \pm 19.51$ & $70.06 \pm 15.79$ & $45.74 \pm 8.63$ & 0.0004 \\
\hline $\begin{array}{l}\text { LAVi }(\mathrm{ml} / \\
\left.\mathrm{m}^{2}\right)^{*}, * * *\end{array}$ & $23.59 \pm 7.09$ & $37.70 \pm 8.89$ & $23.57 \pm 3.80$ & 0.0003 \\
\hline $\mathrm{RA}$ area $\left(\mathrm{cm}^{2}\right)$ & $14.72 \pm 3.80$ & $16.58 \pm 4.85$ & $14.32 \pm 3.33$ & 0.389 \\
\hline $\mathrm{RV}$ area $\left(\mathrm{cm}^{2}\right)$ & $26.09 \pm 4.29$ & $23.15 \pm 4.40$ & $24.37 \pm 3.58$ & 0.238 \\
\hline IVS (mm) & $9.39 \pm 1.41$ & $10.17 \pm 1.26$ & $9.13 \pm 2.04$ & 0.188 \\
\hline PW (mm) & $9.15 \pm 1.31$ & $9.53 \pm 1.33$ & $8.59 \pm 1.98$ & 0.327 \\
\hline LVMi $\left(\mathrm{gr} / \mathrm{m}^{2}\right)$ & $97.56 \pm 17.86$ & $105.40 \pm 21.91$ & $93.23+25.11$ & 0.136 \\
\hline $\begin{array}{l}\text { LVED volume } \\
(\mathrm{ml})^{*}\end{array}$ & $107.1 \pm 26.53$ & $133.8 \pm 39.71$ & $99.54 \pm 19.46$ & 0.043 \\
\hline $\begin{array}{l}\text { LVES volume } \\
(\mathrm{ml})\end{array}$ & $43.45 \pm 13.69$ & $50.57 \pm 18.49$ & $38.21 \pm 10.47$ & 0.055 \\
\hline LVEF \% & $60.21 \pm 6.87$ & $63.36 \pm 7.00$ & $61.81 \pm 5.99$ & 0.567 \\
\hline $\mathrm{CO}(\mathrm{L} / \mathrm{min})^{*}$ & $4.80 \pm 0.86$ & $6.33 \pm 1.28$ & $4.30 \pm 0.70$ & $<0.0001$ \\
\hline $\mathrm{E} / \mathrm{A}$ & $1.29 \pm 0.38$ & $1.40 \pm 0.32$ & $1.38 \pm 0.33$ & 0.442 \\
\hline $\begin{array}{l}\mathrm{e}^{\prime}(\mathrm{cm} / \mathrm{s}) \text { lateral } \\
\text { mitral annulus }\end{array}$ & $13.08 \pm 1.94$ & $11.54 \pm 2.91$ & $12.63 \pm 2.67$ & 0.316 \\
\hline $\mathrm{E} / \mathrm{e}^{3 * * *}$ & $6.01 \pm 1.19$ & $8.27 \pm 2.32$ & $6.38 \pm 1.56$ & 0.025 \\
\hline MPI & $0.31 \pm 0.08$ & $0.32 \pm 0.09$ & $0.27 \pm 0.11$ & 0.502 \\
\hline $\begin{array}{l}\text { QT interval } \\
\text { (msec) }^{*}\end{array}$ & $414.50 \pm 19.19$ & $436.20 \pm 21.95$ & $396.20 \pm 16.58$ & $<0.0001$ \\
\hline
\end{tabular}

\#Kruskal-Wallis test, ${ }^{*}$ CTP B+C vs control, ${ }^{* *}$ CTP A vs control, ${ }^{* * *}$ CTP A vs CTP B+C

MELD score: model for end-stage liver disease, Nt-proBNP: N-terminal propeptide of the brain natriuretic peptide, LAVi: left atrial volume index, RA: right atrium, RV: right ventricle, IVS: interventricular septum, PW: posterior wall, LVMi: left ventricular mass index, LVED: left ventricular end-diastolic, LVES: left ventricular end-systolic, LVEF: left ventricular ejection fraction, CO: cardiac output, MPI: myocardial performance index

\section{DISCUSSION}

Cirrhotic patients have hyperdynamic circulation characterized by increased cardiac output, decreased peripheral vascular resistance and increased plasma volume $[1,23]$. We observed hyperkinetic circulation with increased CO and LVED volume in decompanseted cirrhotic patients. In this patient group, diastolic blood pressure decreased probably due to lowered peripheral resistance [23]. Decreased peripheral resistance and low blood pressure may activate renin angiotensin and aldosterone system which may further increase renal sodium and water reabsorption and intravascular volume. Angiotensin and aldosterone have been implicated in collagen accumulation in myocardial interstitium, myocardial hypertrophy and fibrosis [1]. Indeed, in our cirrhotic patients we observed statistically nonsignificant increase in LVMi. Echocardiographic parameters showing diastolic dysfunction were all in normal limits except for 3 patients. In advanced stages of cirrhosis LAVi increased significantly and although it was within normal limits, E/e' ratio also increased. Wiese et al., also showed a nonsignificant increase in the E/e'ratio with a-24 month follow-up of cirrhotic patients [24]. It was reported that transmitral E/e' ratio correlated well with left ventricular end diastolic filling pressure [25]. Increased LAVi and E/e' ratio are well known indicators of myocardial stiffness and developing diastolic dysfunction. In the literature, diastolic dysfunction was most prominent in patients with severe hepatic fibrosis and decompansation [4] and in these patients the combination of myocardial hypertrophy, diastolic dysfunction [6] and increased heart volumes may lead to cirrhotic cardiomyopathy.

In our study, left ventricular systolic function was normal in patient group when compared with controls. Barbosa et al., showed that $38 \%$ the patients with normal systolic function at rest developed systolic dysfunction during dobutamine infusion and the etiology of cirrhosis was alcohol in $77 \%$ of the patients [26].This may either indicate that classical echocardiographic examination is not sufficient enough to reveal mild systolic dysfunction and pharmacological stress can easily unmask left ventricular contractile problems or myocardial toxic effects of alcohol may have a significant role in decreased contractile reserve during dobutamine infusion. Similarly, Pimenta et al., found that $\mathrm{CO}$ measured by impedance cardiography decreased in patients with cirrhosis, $63 \%$ of whom were alcoholic [11]. In another study, conducted by Saner et al., 10 patients developed systolic dysfunction after liver transplantation and 2 patients known to use alcohol died due to congestive heart failure. Again, in this study, the main cause of cirrhosis in $29.9 \%$ of the patients was alcohol consumption [27].

The MPI has been shown to be useful in evaluating left ventricular function and prognosis of patients with heartrelated disorders. The MPI, reflecting both the global systolic and diastolic cardiac function [12] was not higher in our cirrhotic patients than in controls. The most important reason for this was that our patients with a mean MELD score of 12.7 were at early stages of cirrhosis and none of them exhibited left ventricular systolic dysfunction. Only three of them had diastolic cardiac dysfunction. Song et al., showed that the MPI prior to transjugular intrahepatic portosystemic shunt (TIPS) insertion was high in their cirrhotic patients whose baseline MELD scores were 22 and both pre-TIPS MPI and MELD scores were significantly associated with survival rate after TIPS [16]. 
Wang et al., found that left and right ventricular MPIs increased and left and right ventricular stroke volumes decreased in compensated and decompansated cirrhotic patients [10]. Cirrhotic patients have usually hyperdynamic circulation with increased stroke volumes and cardiac output. In that study, MPI measurement was performed by conventional pulse Doppler examination which might have some limitations. As the echocardiographic data was derived from at least two different cardiac cycles in the conventional method, it could be affected by heart rate variability. However, in PW-TDI one single cardiac cycle is sufficient for Doppler examination. Additionally, Wang et al., did not mention whether patients with alcohol dependance were included in their study or not. Alcoholic cardiomyopathy is different from cirrhotic cardiomyopathy with dilated ventricules and depressed cardiac function [2]. These methodological differences might be the reason of increased MPI in their cirrhotic patients. Similarly, Ashmawy et al., demonstrated that left ventricular ejection fraction decreased and MPI increased in their cirrhotic patients [15]. Unfortunately, they did not report any information about the etiology of cirrhosis in their patient groups. In children whose etiology of cirrhosis were different from alcohol, Amoozgar et al., found that only right ventricular MPI increased before and six months after liver transplantation [17] and Fattouh et al., showed that both left and right ventricular MPIs increased [14].

Nt-pro-BNP is a sensitive marker to detect left ventricular dysfunction and appears to be an important predictor of cardiac complications [3]. In our study, increased intraventricular volume might lead to increased ventricular wall stress and this could stimulate Nt-proBNP secretion from ventricular myocytes. Since, our patients had no past history of cardiovascular disease and alcohol abuse, the increase in Nt-proBNP levels in decompensated patients might be the result of developing diastolic dysfunction and myocardial stress caused by cirrhosis.

In our study, cirrhotic patients showed elevated plasma concentrations of Nt-proBNP which was positively correlated with MELD scores, increased QT duration, CO and right atrial area and negatively correlated with diastolic blood pressure.

In the literature, there was a significant increase in the BNP levels in cirrhotic patients and its levels correlated with the severity of liver disease, CTP score [4, 7-10, 23] and MELD score [27, 28]. Decompensated cirrhotic patients with portal hypertension, ascites, spontaneous bacterial peritonitis, variceal bleeding and hepatic encephalopathy had higher levels of BNP [4-8]. BNP levels were significantly associated with increased left atrial enlargement, left ventricular hypertrophy, cardiac diastolic $[4,5$, $29]$ and systolic dysfunction $[11,26]$.

Hurst et al., found that mean Nt-proBNP levels were $60.8 \pm$ $54.9 \mathrm{pg} / \mathrm{ml}$ and $55.4 \pm 41.4 \mathrm{pg} / \mathrm{ml}$ in patients with mild and moderate liver fibrosis and a cutoff value of greater than $290 \mathrm{pg} /$ $\mathrm{ml}$ was highly predictive of advanced left ventricular diastolic dysfunction [4]. Similarly, in our cirrhotic patients who were in the early stages of cirrhosis, mean Nt-proBNP levels were 63.92 $\pm 18.45 \mathrm{pg} / \mathrm{ml}$. Only 3 patients showed diastolic dysfunction; one patient (CTP A) had mild, one patient (CTP B) had moderate and one patient (CTP B) had severe diastolic dysfunction.
Nt-proBNP levels were found to be negatively related to diastolic blood pressure in this study. In cirrhotic patients low peripheral vascular resistance may lead to decreased blood pressure [1] and with the progression of the disease, while blood pressure decreases the level of Nt-proBNP increases. Similarly, Hurst et al., found a negative relation between blood pressure and NtproBNP levels which increased mainly due to LV diastolic dysfunction [4]. In addition, Henriksen et al., showed a negative correlation between mean arterial blood pressure and proBNP levels [23]. According to Wiese et al., low mean arterial pressure was the only predictor of poor survival in stable cirrhotic patients [24].

In contrast to our findings, no positive relation of proBNP levels to cardiac output was observed in the study conducted by Henriksen et al. [23]. As all patients in their study, had a history of alcohol abuse, it was again impossible to exclude the cardiodepressant toxic effects of alcohol on left ventricular function.

In our study, there was a positive correlation between QT interval and Nt-proBNP levels and QT interval duration was longer in decompensated patients. Similarly, Bernardi et al., showed that prevalence of prolonged QT interval increased as CTP score increased and QT interval prolongation was significantly related to the severity of the underlying liver disease and CTP score. Additionally, they demonstrated a correlation between QT interval duration and plasma norepinephrine levels, indicating that one reason of this electrophysiological abnormality might be the sympathetic overactivity [30]. In the literature other reasons of prolonged QT interval were plasma membrane changes, reduced $\beta$-adrenoceptor density of myocytes, cardiotoxic effects of bile salts and nitric oxide [31].

Nt-proBNP levels were found to be positively correlated also with RA area in our study. Zhang et al., found that in alcoholic cirrhotic patients RA maximal area and RA volume index were higher and both RA and RV functions deteriorated. Unfortunately, in that study the influence of alcohol on right heart functions could not be excluded [32]. Patients with increased RA pressure before and after transjugular intrahepatic portosystemic shunt (TIPS) had approximately twice the mortality of those with normal RA pressure [33]. In the study of Fattouh et al., cirrhotic children had dilated RV and their $\mathrm{BNP}$ levels were correlated with $\mathrm{E} / \mathrm{e}^{\prime}$ ratio of $\mathrm{RV}$, indicating that right heart functions were even affected in cirrhotic children [14]. Right ventricular diastolic dysfunction rate was reported to be high in chronic liver diseases [34], explaining positive correlation between RA area and Nt-proBNP levels.

Here, we did not observe systolic dysfunction and increased MPI in nonalcoholic cirrhotic patients. However, we demonstrated that in early stages of cirrrhosis increased Nt -proBNP levels together with diastolic dysfunction were positively correlated with MELD score, QT duration, RA area, CO and were negatively corelated with diastolic blood pressure. It is possible that in the late stages of disease, systolic dysfunction may develop and MPI may increase but this would better be evaluated further in studies especially addressing nonalcoholic patients. 


\section{Compliance with the Ethical Standards}

Ethical Approval: The study was approved by the Clinical Research Ethics Committee of Marmara University, School of Medicine, Istanbul (03 - 2006-0124), and informed written consent was obtained from all the participants. The study was conducted in accordance with the principles of the Declaration of Helsinki.

Financial Support: The authors have no relevant financial information to close.

Conflict of Interest: The authors have no potential conflicts of interest to disclose

Authors' Contributions: STT, ASF and OO: Drafting of the work, STT and OO: Concept and design of the study, STT and FYE: Data acquisition, STT and OO: Statistical analysis, STT and FE: Medical practices. All authors critically revised the manuscript, approved the final version to be published, and agreed to be accountable for all aspects of the work.

\section{REFERENCES}

[1] Møller S, Danielsen KV, Wiese S, Hove JD, Bendtsen F. An update on cirrhotic cardiomyopathy. Expert Rev Gastroenterol Hepatol 2019; 13: 497-505. doi: 10.1080/17474.124.2019.1587293.

[2] Mirijello A, Tarli C, Vassallo GA, et al. Alcoholic cardiomyopathy: What is known and what is not known. Eur J Intern Med 2017;43: 1-5. doi: 10.1016/j.ejim.2017.06.014.

[3] Rubattu S, Sciarretta S, Valenti V, Stanzione R, Volpe M. Natriuretic peptides: an update on bioactivity, potential therapeutic use, and implication in cardiovascular diseases. Am J Hypertens 2008; 21: 733-41. doi: 10.1038/ajh.2008.174.

[4] Raedle-Hurst TM, Welsch C, Forestier N, et al. Validity of $\mathrm{N}$-terminal propeptide of the brain natriuretic peptide in predicting left ventricular diastolic dysfunction diagnosed by tissue Doppler imaging in patients with chronic liver disease. Eur J Gastroenterol Hepatol 2008; 20: 865-73. doi: 10.1097/ MEG.0b013e3282fb7cd0.

[5] Wong F, Siu S, Liu P, Blendis LM. Brain natriuretic peptide: is it a predictor of cardiomyopathy in cirrhosis? Clinical Science (Lond) 2001; 101: 621-8.

[6] Abbas WA, Kasem Ahmed SM, Abdel Aal AM, et al. Galactin-3 and brain natriuretic peptide versus conventional echocardiography in the early detection of cirrhotic cardiomyopathy. Turk J Gastroenterol 2016; 27: 367-74. doi: 10.5152/tjg.2016.16100.

[7] Yildiz R, Yildirim B, Karincaoglu M, Harputluoglu M, Hilmioglu F. Brain natriuretic peptide and severity of disease in non-alcoholic cirrhotic patients. J Gastroenterol Hepatol 2005; 20: 1115-20. doi: 10.1111/j.1440-1746.2005.03906.x.

[8] Metwaly A, Khalik AA, Nasr FM, Sabry AI, Gouda MF, Hassan M. Brain natriuretic peptide in liver cirrhosis and fatty liver:
Correlation with cardiac performance. Electron Physician 2016; 8:1984-93. doi: 10.19082/1984.

[9] Shi LY, Jin R, Lin CJ, et al. B-type natriuretic peptide and cirrhosis progression. Genet Mol Res 2015; 14: 5188-96. doi: 10.4238/2015.May.18.9.

[10] Wang LK, An XF, Wu XL et al. Doppler myocardial performance index combined with plasma B-type natriuretic peptide levels as a marker of cardiac function in patients with decompensated cirrhosis. Medicine (Baltimore) 2018; 97: e13302. doi: 10.1097/MD.000.000.0000013302.

[11] Pimenta J, Paulo C, Gomes A, Silva S, Rocha-Gonçalves F, Bettencourt P. B-type natriuretic peptide is related to cardiac function and prognosis in hospitalized patients with decompensated cirrhosis. Liver Int 2010; 30: 1059-66. doi: 10.1111/j.1478-3231.2010.02266.x.

[12] Pellett AA, Tolar WG, Merwin DG, Kerut EK. The Tei index: methodology and disease state values. Echocardiography 2004; 21: 669-72. doi: 10.1111/j.0742-2822.2004.04052.x.

[13] Gaibazzi N, Petrucci N, Ziacchi V. Left ventricle myocardial performance index derived either by conventional method or mitral annulus tissue-Doppler: a comparison study in healthy subjects and subjects with heart failure. J Am Soc Echocardiogr 2005; 18: 1270-6. doi: 10.1016/j.echo.2005.06.006.

[14] Fattouh AM, El-Shabrawi MH, Mahmoud EH, Ahmed WO. Evaluation of cardiac functions of cirrhotic children using serum brain natriuretic peptide and tissue Doppler imaging. Ann Pediatr Cardiol 2016; 9: 22-8. doi: 10.4103/09742069.171373.

[15] Ashmawy MM, Younis HA, Elbaset MAA, et al. Evaluation of cardiac function in patients with liver cirrhosis using tissue Doppler study. Egypt J Intern Med 2018; 30: 115-20. doi:10.4103/ejim.ejim_28_18.

[16] Song Y, Li W, Xue H, Ruan L. Tei index is associated with survival in cirrhosis patients treated with transjugular intrahepatic portosystemic shunt. Echocardiography 2019; 36: 61-66. doi: 10.1111/echo.14201.

[17] Amoozgar H, Ermis R, Honar N, Malek-Hosseini SA. Myocardial performance after successful liver transplantation. Int J Organ Transplant Med 2016; 7: 77-83.

[18] Lang RM, Badano LP, Mor-Avi V, et al. Recommendations for cardiac chamber quantification by echocardiography in adults: an update from the American Society of Echocardiography and the European Association of Cardiovascular Imaging. J Am Soc Echocardiogr 2015; 28:1-39.e14. doi: 10.1016/j. echo.2014.10.003.

[19] Nagueh SF, Smiseth OA, Appleton CP, et al. Recommendations for the evaluation of left ventricular diastolic function by echocardiography: An update from the American Society of Echocardiography and the European Association of Cardiovascular Imaging. J Am Soc Echocardiogr 2016; 29 : 277-314. doi: 10.1016/j.echo.2016.01.011.

[20] Armstrong WF, Ryan T. Feigenbaum's Echocardiography. 8th ed. Hemodynamics Chapter 8. Philadelphia: Lippincott Williams and Wilkins, 2018:648-9. 
[21] Su HM, Lin TH, Voon WC, et al. Differentiation of left ventricular diastolic dysfunction, identification of pseudonormal/restrictive mitral inflow pattern and determination of left ventricular filling pressure by Tei index obtained from tissue Doppler echocardiography. Echocardiography 2006; 23: 287-94. doi: 10.1111/j.15408175.2006.00222.x.

[22] Nayor M, Cooper LL, Enserro DM, et al. Left ventricular diastolic dysfunction in the community: Impact of diagnostic criteria on the burden, correlates, and prognosis. J Am Heart Assoc 2018; 7: e008291. doi: 10.1161/JAHA.117.008291.

[23] Henriksen JH, Gøtze JP, Fuglsang S, Christensen E, Bendtsen F, Møller S. Increased circulating pro-brain natriuretic peptide (proBNP) and brain natriuretic peptide (BNP) in patients with cirrhosis: relation to cardiovascular dysfunction and severity of disease. Gut 2003; 52:1511-7. doi: 10.1136/gut.52.10.1511.

[24] Wiese S, Hove JD, Mo S, et al. Cardiac dysfunction in cirrhosis: a 2-yr longitudinal follow-up study using advanced cardiac imaging. Am J Physiol Gastrointest Liver Physiol 2019; 317: G253-G263. doi: 10.1152/ajpgi.00402.2018.

[25] Nagueh SF, Mikati I, Kopelen HA, Middleton KJ, Quiñones MA, Zoghbi WA. Doppler estimation of left ventricular filling pressure in sinus tachycardia. A new application of tissue doppler imaging. Circulation 1998; 98:1644-50. doi: 10.1161/01.cir.98.16.1644.

[26] Barbosa M, Guardado J, Marinho C, et al. Cirrhotic cardiomyopathy: Isn't stress evaluation always required for the diagnosis? World J Hepatol 2016; 8: 200-6. doi: 10.4254/wjh. v8.i3.200.

[27] Saner FH, Neumann T, Canbay A, et al. High brainnatriuretic peptide level predicts cirrhotic cardiomyopathy in liver transplant patients. Transpl Int 2011; 24: 425-32. doi: 10.1111/j.1432-2277.2011.01219.x.

[28] Padillo J, Rioja P, Muñoz-Villanueva MC, et al. BNP as marker of heart dysfunction in patients with liver cirrhosis. Eur J Gastroenterol Hepatol 2010; 22:1331-6. doi: 10.1097/ MEG.0b013e32833e6b2a.

[29] Falletta C, Filì D, Nugara C, et al. Diastolic dysfunction diagnosed by tissue Doppler imaging in cirrhotic patients: Prevalence and its possible relationship with clinical outcome. Eur J Intern Med 2015; 26: 830-4. doi: 10.1016/j. ejim.2015.10.009.

[30] Bernardi M, Calandra S, Colantoni A, et al. Q-T interval prolongation in cirrhosis: prevalence, relationship with severity, and etiology of the disease and possible pathogenetic factors. Hepatology 1998; 27: 28-34. doi: 10.1002/ hep.510270106.

[31] Zambruni A, Trevisani F, Caraceni P, Bernardi M. Cardiac electrophysiological abnormalities in patients with cirrhosis. J Hepatol 2006; 44: 994-1002. doi: 10.1016/j.jhep.2005.10.034.

[32] Zhang K, Braun A, von Koeckritz F, et al. Right heart remodeling in patients with end-stage alcoholic liver cirrhosis: speckle tracking point of view. J Clin Med 2019; 8:1285. doi: 10.3390/jcm8091285.

[33] Parvinian A, Bui JT, Knuttinen MG, Minocha J, Gaba RC. Right atrial pressure may impact early survival of patients undergoing transjugular intrahepatic portosystemic shunt creation. Ann Hepatol 2014;13: 411-9. doi:10.1016/S16652681(19)30848-8.

[34] Karabulut A, Iltumur K, Yalcin K, Toprak N. Hepatopulmonary syndrome and right ventricular diastolic functions: an echocardiographic examination. Echocardiography 2006 23: 271-8. doi: 10.1111/j.1540-8175.2006.00210.x. 